manifestations of systemic LE (SLE). The typical histopathologic pattern in CLE/SLE is interface dermatitis, which can also be observed in dermatomyositis (DM). While LE may affect any organ system, DM most commonly affect muscles and skin.

The aim of this study was to investigate the whole proteome of skin inflammatory foci in the cohort of CLE and DM patients in a comparative, hypothesis-free manner and identify disease-unique molecular mechanisms.

Methods CLE $(n=6)$, DM $(n=5)$ patients and controls $(n=6)$ were recruited at diagnosis or disease exacerbation. Skin biopsies were acquired, examined by a pathologist and selected inflammatory foci were laser micro-dissected. The total protein content was analyzed by mass-spectrometry, further analysis was performed by string-db.org platform. Certain proteomic findings were confirmed by immunohistochemistry (IHC).

Results CLE infiltrates were more protein rich in comparison to DM lesions. There ratio of $5 \mathrm{x}$ upregulated proteins in LE/ $\mathrm{DM}$ was 60 , while ratio for $\mathrm{DM} / \mathrm{LE}$ was 13 . Our results confirmed high abundance of (IFN)-regulated proteins both in CLE and DM, including: IFIT1, IFIT2, IFIT3, MX1, MX2, OAS2, OAS3, STAT1,- STAT2, DDX58, DDX60 and EIF2AK2. Proteins expressed differentially in CLE covered complement proteins (C1b), including membrane attack complex (MAC) (C5, C6, C7, C8A and B) and complement regulators (CFHR1, CFHR2, CFHR5), as well as regulators of coagulation: thrombospondin 2 (THBS2), thrombin (F2), fibrinogen (F12) and annexin A3 (ANXA3). Importantly, we identified interleukin (IL) -16 as the only detectable and highly abundant cytokine in the CLE lesions and confirmed this finding by IHC.

Conclusions Our data confirm evidence on IFN-regulated processes in CLE/SLE. Importantly, we identified IL-16 as a novel cytokine most strongly upregulated locally in the skin lesions. Moreover, we identified activation of MAC, complement regulating proteins as well as involvement of coagulation/fibrinolysis system. The study brings information on novel pathways involved in the inflammatory foci of the skin lesions in CLE patients. Our findings are of interest in further search of new therapeutic targets.

\section{O33 PER-PROTOCOL REPEAT KIDNEY BIOPSY PORTENDS RELAPSE AND LONG-TERM OUTCOME IN INCIDENT CASES OF PROLIFERATIVE LUPUS NEPHRITIS}

${ }^{1}$ Ioannis Parodis, ${ }^{2}$ Christina Adamichou, ${ }^{3}$ Selda Aydin, ${ }^{1}$ Alvaro Gomez, ${ }^{4}$ Nathalie Demoulin,
${ }^{5}$ Julia Weinmann-Menke, ${ }^{2}$ Frédéric A Houssiau, ${ }^{2}$ Farah Tamirou. ${ }^{1}$ Dept. of Medicine Solna,
Karolinska Institutet and Rheumatology, Karolinska University Hospital, Stockholm, Sweden;
${ }^{2}$ Rheumatology Dept., Cliniques Universitaires Saint-Luc and Pôle de Pathologies
Rhumatismales Inflammatoires et Systémiques, Institut de Recherche Expérimentale et
Clinique, Université catholique de Louvain, Brussels; ${ }^{3}$ Pathology Dept., Cliniques
Universitaires Saint-Luc, Brussels; ${ }^{4}$ Division of Nephrology, Cliniques Universitaires Saint-Luc
and Institut de Recherche Expérimentale et Clinique, Université catholique de Louvain,
Brussels, Belgium; ${ }^{5}$ Dept. of Nephrology, Rheumatology and Clinical Immunology, University
Medical Centre of the Johannes Gutenberg-University Mainz, Mainz, Germany

10.1136/lupus-2020-eurolupus.44

Background In patients with Lupus Nephritis (LN), clinical response to treatment and renal histopathology have been shown to be discordant. We investigated whether per-protocol repeat renal biopsies are predictive of $\mathrm{LN}$ relapses and longterm impairment of renal function.
Methods Forty-two patients with an incident biopsy-proven active proliferative (class III/IV $\pm \mathrm{V}$ ) $\mathrm{LN}$ from the $\mathrm{LN}$ database of the Université catholique de Louvain were included in the present retrospective study. Per-protocol repeat kidney biopsies were performed in all patients after a median time of 24.3 (IQR: 21.3-26.2) months. The NIH activity index (AI) and chronicity index (CI) scores were assessed in both baseline and repeat biopsies.

Results Despite a moderate correlation between urinary protein/creatinine (U-P/C) ratios and AI scores at repeat biopsy $(\mathrm{r}=0.48 ; \mathrm{P}=0.001)$, ten patients $(23.8 \%)$ with $\mathrm{U}-\mathrm{P} / \mathrm{C}$ ratios $<1.0 \mathrm{~g} / \mathrm{g}$ still had a high degree of histological activity (AI score $>3$; figure 1). High AI scores in repeat biopsies were associated with an increased probability and/or shorter time to renal relapse $(\mathrm{N}=11)$ following the repeat biopsy (HR: 1.2; 95\% CI: 1.1-1.3; P=0.007), independently of proteinuria levels. High NIH CI scores in repeat biopsies were associated with a sustained increase in serum creatinine levels corresponding to $\geq 120 \%$ of the baseline value (HR: $1.8 ; 95 \% \mathrm{CI}$ : 1.1-2.9; $\mathrm{P}=0.016)$ through a median follow-up time of 131.5 (IQR: 73.8-178.2) months. Baseline AI/CI scores were not predictive of these outcomes.

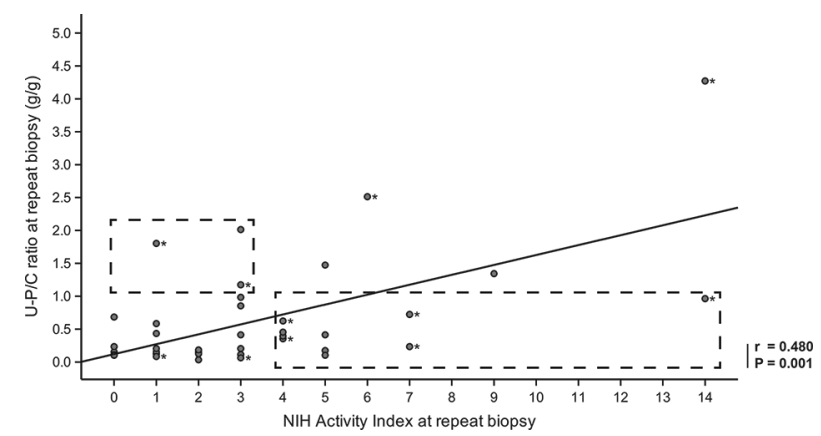

Abstract 033 Figure 1

Conclusions Our results highlight the usefulness of per-protocol repeat biopsies as an integral part of the treatment evaluation, also in patients who have shown adequate clinical response.

\section{VARIANTS IN BANK1 ARE ASSOCIATED WITH LUPUS NEPHRITIS}

${ }^{1}$ Karin Bolin, 'Dag Leonard, 'Johanna K Sandling, 'Andrei Alexsson, 'Pascal Pucholt, ${ }^{2}$ Malena Loberg Haarhaus, ${ }^{3}$ Joanne Nititham, ${ }^{4}$ Andreas Jönsen, ${ }^{5}$ Christopher Sjöwall, ${ }^{4}$ Anders A Bengtsson, ${ }^{6}$ Solbritt Rantapää-Dahlqvist, ${ }^{2}$ Elisabet Svenungsson, ${ }^{2}$ Iva Gunnarsson, ${ }^{1}$ Ann-Christine Syvänen, ${ }^{7}$ Karoline Lerang, ${ }^{8}$ Anne Troldborg, ${ }^{9}$ Anne Voss, ${ }^{7}$ Øyvind Molberg, ${ }^{10}$ Søren Jacobsen, ${ }^{3}$ Lindsey Criswell, 'Lars Rönnblom, 'Gunnel Nordmark. ${ }^{1}$ Uppsala University, Uppsala; ${ }^{2}$ Karolinska Institutet, Solna, Sweden; ${ }^{3}$ University of California, San Francisco, USA; ${ }^{4}$ Lund University, Lund; ${ }^{5}$ Linköping University, Linköping; ${ }^{6}$ Umeå University, Umeå, Sweden; 7 University of Oslo, Oslo, Norway; ${ }^{8}$ Aarhus University, Aarhus; ${ }^{9}$ Odense University Hospital, Odense; ${ }^{10}$ Copenhagen University Hospital, Copenhagen, Denmark

\subsection{6/lupus-2020-eurolupus.45}

Background Lupus Nephritis (LN) is a cause of significant morbidity in SLE. While the genetic background to SLE has been well characterized, less is known about genes predisposing to $\mathrm{LN}$. 